\title{
Mathematical modeling of emergency situations at objects of production and gas transportation
}

\author{
Victor Orlov*, Elena Detina and Oleg Kovalchuk \\ Moscow State University of Civil Engineering, 26, Yaroslavskoe Shosse, 129337 Moscow, Russia
}

\begin{abstract}
Mathematical modeling attracts the attention of researchers from different fields, as one of the rigorous methods of justifying the conducted researchs. Mathematical modeling makes it possible forecast to real life situations. Based on the mathematical model, the work provides the innovative recommendations for making managerial decisions on increasing the reliability and functional safety of the pipeline at all stages of its life cycle. The results obtained allow: 1) to consider issues related to the rational management of resources for the maintenance of the infrastructure of gas chemical complexes in conditions of limited means; 2) is have adapt RAMS methodology to the complex of Russian standards and the base of normative and methodological documentation on managing the life cycle processes of systems of to product and transportat of gas.
\end{abstract}

\section{Introduction}

Currently, the leading organizations of production and processing of oil, gas and related industries of the European Union are implementing the life-cycle management system of the facility in accordance with the international standard API RP 581: 2016. [1]

The risk assessment methodology (RBI) is widely used internationally, especially in the oil and gas industry, and is well known in France. The literature [1] reports on several RBI projects demonstrating the strengths of the approach to risk management and optimizing inspection schedules. API RP 581 is a well-proven methodology for conducting RBI in the processing industry, and the 3rd edition of the standard was published only in April 2016. This article examines the new features of the 3rd edition, in particular for internal and external cracks and corrosion, and also discusses a case study of the application of this latest RBI methodology in France. This is happening in the context of the European inspection service operating in accordance with the French regulatory framework. The document is intended for the ubiquitous application of the RBI methodology. In the context, when planning for site inspections should combine risk calculations with regulatory constraints. The quantitative approach of API RP 581 is a good basis for optimized, sequential and "automatic" planning of inspections and can be performed to work in accordance with the current rules.

This system allows to ensure the operation of gas production and transportation facilities in conditions of limited financial resources, where the main goal is to achieve a

\footnotetext{
Corresponding author: OrlovVN@mgsu.ru
} 
balance between costs, risks and required asset performance, but the lack of this system is the inability to obtain predicted values of undesirable situations at gas production and transportation facilities. We draw attention to the fact that the importance of risk from the point of view of management is not determined by one parameter, which is related to its probabilistic nature. Obviously, the risk, which in the case of implementation carries a large financial loss, can be considered dangerous and requiring management. But if the probability of this risk is extremely small, then they can be neglected. Accordingly, and vice versa: a risk with a small potential loss, but realized very often, eventually lead to a significant total financial damage. Therefore, to characterize each identified risk is necessary with the help of its two main parameters: the probability of implementation and the magnitude of possible financial damage.

It should be noted that the consequences of the implementation of risks are not only financial, but also accompanied by loss of life and health of people, so it is desirable in Russian existing methodologies, similar to the foreign option, to translate these indicators into financial ones. This is due to the fact that in economic activity it is this kind of damage indicators that are of greatest importance.

Thus, each identified risk, if assessed, will be characterized by two values: the probability of its occurrence and the amount of financial losses.

\section{Methodology}

The existing methodology for assessing risks abroad is based on statistical information of enterprises of oil and gas chemical complexes and has only ascertaining the nature of the event. In our variant, it is proposed to eliminate the above mentioned shortcoming to the foreign risk assessment system: at the first stage, it should be supplemented with a mathematical model that allows forecasting emergency situations in the continuation of the life cycle of the facility. Forecasting gives us important information about the nature of the trend of emergency situations, which makes it possible to evaluate the effectiveness of measures taken to reduce the number of emergency situations; at the second stage, based on the probabilistic approach in the study of the causes of emergencies at the gas pipeline facilities, to develop an algorithm for affecting the trend component in order to prevent emergencies. In this paper we present the solution of the first stage.

The scientific publications published in fundamental research [2, 3], construction [4-6], cattle breeding $[7,8]$, medicine $[9,10]$, education $[11,12]$, in economy [13]. For the construction of the mathematical model, information from the reporting documentation on emergency situations at the production and transportation facilities of Rostekhnadzor gas was used. [14] Statistical information of emergency situations on the pipeline reflects implicitly the technical state of the production process of gas distribution, and the applied mathematical methods of processing this information allow to take into account the technical state of the production process of gas distribution when obtaining the predicted result. Let us recall the definitions of the basic concepts of mathematical modelling. [15]

Definition 1. A model is an image or reflection of a process or phenomenon obtained with the help of special tools.

Special means are classified into: mathematical, technical, computer, etc. Thus, on the basis of the classification of special means, we obtain the necessary definitions of models.

Definition 2. A mathematical model is an image or reflection of a process or phenomenon, obtained by means of mathematical means.

Based on the statistics of emergency situations on gas pipelines from 2012 to 2017. [14] 


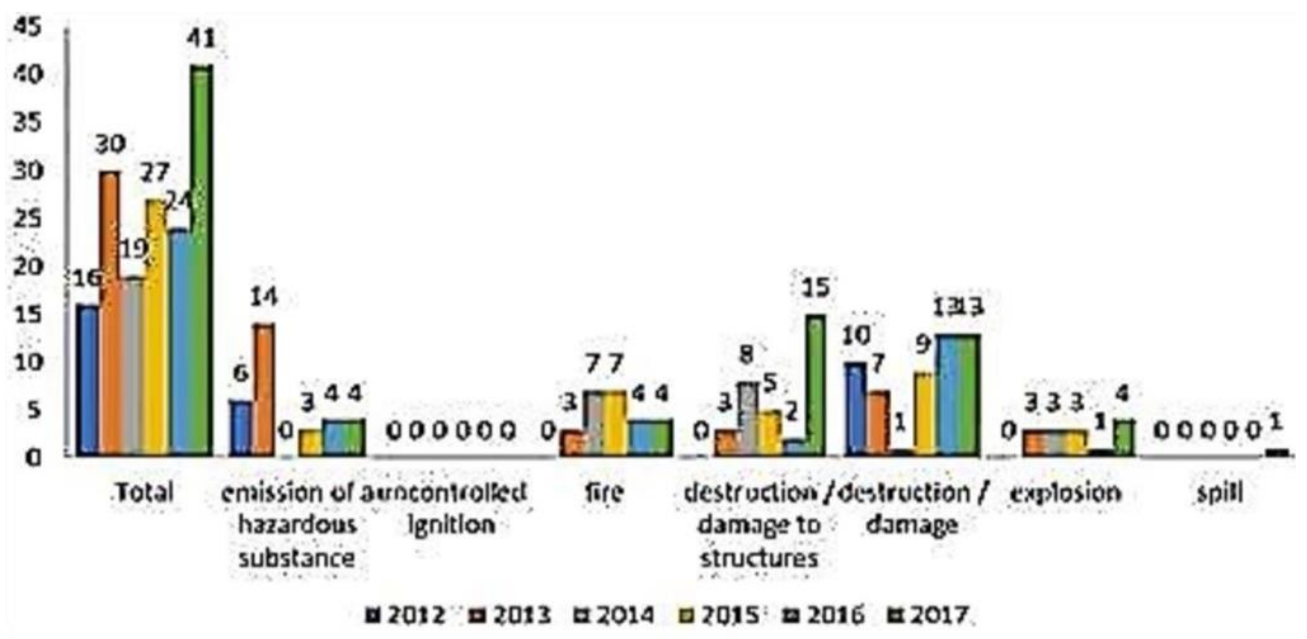

Fig. 1. Statistics of emergency situations on gas pipelines from 2012 to 2017.

A mathematical model is constructed. The available statistical information was considered as a time series for which an additive model was constructed. Structure of the additive model

$$
\mathrm{Y}=\mathrm{S}+\mathrm{T}+\mathrm{E}
$$

where $\mathrm{S}$ is a cyclic component, $\mathrm{T}$ is a trend component, and $\mathrm{E}$ is a random component. The value of Y means the number of emergency situations and is measured in whole units at the final stage of the calculations. The trend component has the form

$$
\mathrm{T}=17.4792+2.4821 \mathrm{t}
$$

where $t$ is an instant of time. The geometric interpretation of the initial information and the constructed model is shown in Fig. 2, where the row 1 corresponds to the initial information, and the series 2 corresponds to the constructed model.

\section{Dynamics of emergency situations from 2012 to 2017}

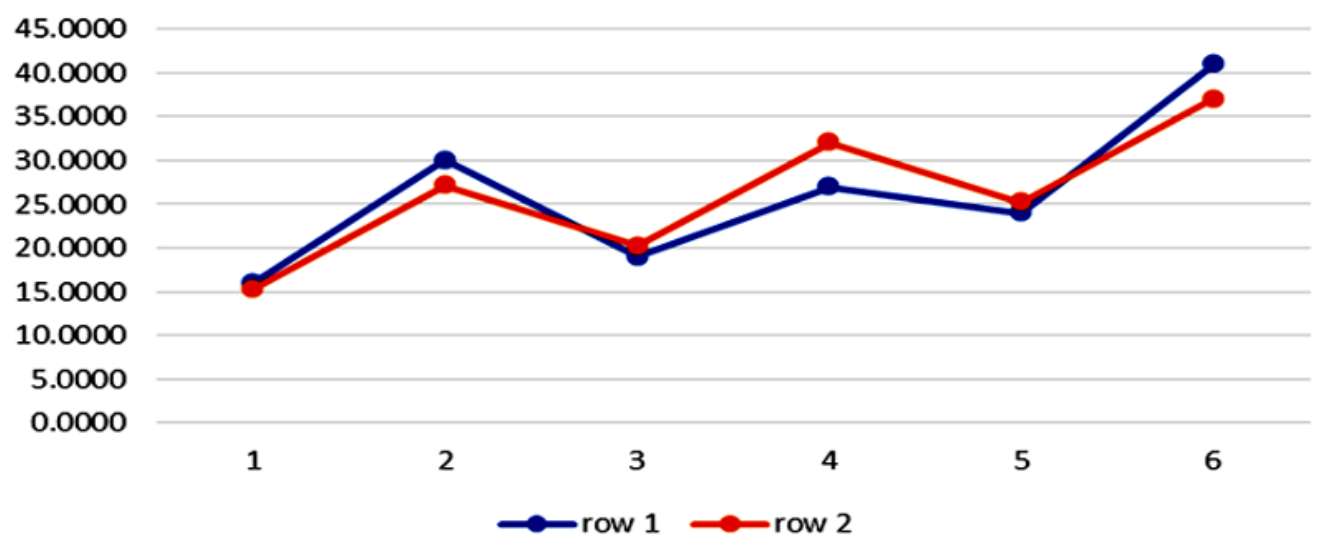

Fig. 2. Dynamics of emergency situations on gas pipelines from 2012 to 2017. 
As a result of the use of the proposed mathematical model and in accordance with the work [15]:

- Based on the variance analysis, the quality indicator of the constructed model was calculated $R^{2}=0,8652$.

- The importance of mathematical model was established using Fisher's statistical criterion $\alpha=0,05 ; \quad F_{к р}=7,71 ; \quad F_{\text {набл }}=25,6721$ and the adoption of a competing hypothesis $H_{1}: R^{2} \neq 0$.

- For 2018, a confidence interval of the predicted value was obtained $26 \leq Y_{2018} \leq 34$.

Thus, after comparison with the values of Fig. 2 it is possible to predict the decline of emergency situations in 2018.

\section{Conclusions}

To ensure the management of risks in the field of security of gas distribution, a mathematical model of the dynamics of the change in emergency situations is constructed at the first stage, which allows: 1) to obtain the predicted result at the next moment of time; 2) determine the nature of the trend of emergency situations; 3 ) determine the effectiveness of the management decision by the nature of the trend component. In general, the first stage is the beginning of the formation of a management decision in the task of safety of production and transportation of gas. The completion of the management decision is connected with the solution of the tasks of the second stage - the development of an algorithm based on the probabilistic approach and allowing to influence the trend component of the constructed mathematical model.

The gas pipeline security management system based on risk assessment will support decision-making, taking into account not only the number of security violations, but also the corresponding damages. Correlation of the magnitude of risks with the cost of measures to improve traffic safety will allow in the conditions of resource constraints to make management decisions that meet economic criteria.

\section{References}

1. API RP 581, Standard by American Petroleum Institute (2016)

2. V.N. Orlov, S.A. Redkozubov, Izvestiya Institute of Engineering Physics J., 4, 18 (2010)

3. V.N. Orlov, O.A. Kovalchuk, IOP Conf. Ser.: Mater. Sci. Eng. 365 (2018)

4. O.A. Kovalchuk, Procedia Engineering J. 153, 304 (2016)

5. O.A. Kovalchuk, PGS J., 9, 70 (2016)

6. O.A. Kovalchuk, PGS J., 11, 53 (2014)

7. N.I. Kul'makova, V.N. Orlov, Bulletin of Kurgan GSKHA, 2, 40 (2016)

8. N.I. Kul'makova, V.N. Orlov, Bulletin of Belgorod GSKHA, 4, 130 (2016)

9. V.N. Orlov, Preventing diseases and promoting health J., 5, 44 (2007)

10. V.N. Orlov, Kazan Medical J., 90, 889 (2009)

11. V.N. Orlov, N.Ye. Pikina, Informatics and Education J., 1, 109 (2008)

12. V.N. Orlov, N.Ye. Pikina, Materials Intl. scientific-practical of Cheboksary (2009)

13. V.N. Orlov, T.V Ivanov, Economics of agricultural and processing enterprises J., 12, 73 (2016) 
14. Information Bulletin of the Federal Service for Environmental, 1, 19 (2018)

15. V.N. Orlov, Bulletin of RGSU, 1, 128 (2014) 